\title{
A hyperelastic deformable template for cardiac segmentation in MRI
}

\author{
Youssef Rouchdy ${ }^{1}$, Jérôme Pousin ${ }^{2}$, Joël Schaerer ${ }^{3}$, Patrick Clarysse ${ }^{3}$ \\ 1 Projet ICARE, INRIA Sophia-Antipolis, 2004 route des lucioles - BP 93 FR-06902 \\ Sophia Antipolis \\ 2 Institut C. Jordan, UMR CNRS 5028, INSA de Lyon, 69621 Villeurbanne cedex, \\ France \\ 3 CREATIS CNRS UMR 5220, INSERM U630, INSA Bât. Blaise Pascal, \\ 69621 Villeurbanne cedex, France
}

\begin{abstract}
This article proposes a hyperelastic 3D deformable template for the segmentation of soft structures. It relies on a template, which is a topological, geometrical and material model of the structure to segment. The template is modeled as an elastic body which is deformed by forces derived from the image. The proposed model is based on the nonlinear three-dimensional elasticity problem with a boundary condition of pure traction. In addition, the applied forces depend on the displacements. For computations, a convergent algorithm is proposed to minimize the global energy of template deformation. A discrete algorithm using the finite element method is presented and illustrated on MR images of mice.
\end{abstract}

\section{Introduction}

Most recent medical imaging systems can provide a great amount of data explaining the anatomy and function of a patient's organs. However, the development of efficient tools for automatic processing is mandatory to fully exploit the wealth of information obtained by medical imaging systems and to provide quantified parameters. The context of this paper is related to the extraction of the heart's anatomy and motion from temporal image sequences, more precisely Magnetic Resonance Imaging (MRI) sequences. Currently, a clinical examination results in a stack of slices covering the whole heart at successive time points over the cardiac cycle. These imaging data constitute the input of the segmentation tracking approach proposed in this paper. The methodology we follow for the segmentation is based on the deformable model principle and, as such, relies on a a priori model of the structure to be segmented [10]. In the great variety of deformable models, our approach uses a volumetric tetrahedral mesh of the heart with elastic properties. We call it Deformable Elastic Template (DET). The linear DET model has been previously introduced in [12], [13] for the segmentation of the heart ventricles. In this paper, we introduce the new nonlinear DET model, which is less sensible to initialization, along with several improvements towards the automatic segmentation of cardiac structures in MR images. 


\section{Related work}

Segmentation and motion estimation of the cardiac structures is one of the most popular applications in medical image analysis. Numerous segmentation techniques have been tested in this context from basic thresholding and low-level methods to more sophisticated modeling and learning approaches [4]. The used methodology and the results depend on the imaging modalities and the number of dimensions (2D, 3D, 2D+time, 3D+time). Up to now, Magnetic Resonance Imaging and Ultrasounds have been mainly addressed for both static and dynamic segmentation. However, the inherent difficulties (image artifacts, noise and motion) are such that no generic method has truly emerged yet for routine practice. It is clear that prior knowledge needs to be taken into account to better constrain the segmentation. Therefore, methods based on a priori models of the heart geometry, known as deformable models, have retained attention and obtained a certain success in practice. The final segmentation results from the minimization of a global energy functional which establishes the balance between an internal energy, constraining the structure's shape, and an external energy, representing the action of image data onto the model. Contour and surface models have been extensively studied for segmenting soft structures. Their extension to shape tracking through $2 \mathrm{D}$ or $3 \mathrm{D}$ image sequences has generally come to the use of the result at time point $t$ as an initialization for the segmentation at time point $t+1$ with some temporal smoothness constraints [6]. The extraction of both endocardial and epicardial cardiac surfaces is considered either as the coupled segmentation of two surfaces [6] or through the introduction of more complex volumetric models [12]. These latter models involve a volumetric representation of the heart associated to behavioral laws, such as elasticity. Level set methods, which can be closely linked with the previous deformable models, have also been investigated in this context [11]. In this particular approach, however, the shape topology is allowed to change during the optimization process which is not always desirable. Another popular approach is based on prior learning or cardiac atlases. The prior model is a summary representation of the manual segmentation of a (great, as big as possible) number of patient data sets. One of the main difficulties is to be able to establish a unique correspondence between all the segmentations to build up the model $[4,7]$. This statistical model then constrains the segmentation of the new data set through active shape (shape only)[5] or active appearance models (taking into account image Grey levels) [9]. Such approaches are very interesting but face the problem of representativity of the database (defined by the number of individuals). It is however clear that prior models of anatomical structures can greatly improve the segmentation. The volumetric deformable model proposed here incorporates both geometry and physics of the cardiac structures.

\section{Hyperelastic model for soft structure segmentation}

A geometric template represents the object's interfaces as well as its interior and the properties assigned to it. The template is first placed within the image close 
to the structure to be extracted. It is then deformed iteratively by applying a force field so that its edges stick to the borders of the targeted structure. Segmentation through template deformation is achieved by the minimization of a global energy (or functional). The global energy is generally composed of two terms. The first term is computed from the image data. Its role is to guide the deformation towards the border of the targeted object. The second term introduces a regularity constraint on the desired deformations. It also ensures that the problem is expressed in a suitable functional space.

The initial Deformable Elastic Template introduced in [12] relied on linear elasticity to deform the template. In this article, the regularization term comes from nonlinear elasticity and allows for large deformations. No part of the geometrical model is maintained fixed during the deformation process, which contributes to the better robustness of the segmentation against model initialization. The equivalence between the minimization problem and local elasticity equations is ensured when the material is hyperelastic and the applied forces are conservative (i.e. derived from a potential energy) [3].

For the considered applications, Saint-Venant Kirchhoff material is considered which is hyperelastic and the simplest model among all nonlinear models. The applied forces are assumed to be conservative. Let $\Omega$ be the domain to be deformed (a bounded subset of $\mathbb{R}^{3}$ ), let $\nu$ be the unit outward normal to $\partial \Omega$ the boundary of $\Omega, u: \bar{\Omega} \rightarrow \mathbb{R}^{3}$ be the displacement and $E$ be the following strain tensor

$$
E(u)=\frac{1}{2}\left(\nabla u^{t}+\nabla u+\nabla u^{t} \nabla u\right),
$$

A Saint-Venant Kirchhoff material is hyperelastic and homogeneous, thus the strain energy is independent of a particular point $x \in \bar{\Omega}$ and defined by the relation

$$
W(x, E)=W(E)=\frac{\lambda}{2}(\operatorname{Tr} E)^{2}+\mu \operatorname{Tr} E^{2}
$$

The internal energy is defined for the reference state $\Omega$ by

$$
E_{\text {int }}(u)=\int_{\Omega} W(\nabla(\mathbf{1}+u)(x)) \mathrm{d} x
$$

while the external energy is expressed for the deformed state as:

$$
E_{\text {ext }}(u)=-\int_{\partial \Omega} \hat{G}(\mathbf{1}+u) d \sigma
$$

The function $\hat{G}$ is the potential of the applied surface forces. When the applied force field is conservative, the minimization of the global energy

$$
E_{\text {total }}(u)=E_{\text {int }}(u)+E_{\text {ext }}(u)
$$

is "formally" equivalent to solving the following Euler equations (see [3]): 


$$
\begin{aligned}
& \operatorname{div}((\mathbf{1}+\nabla u) \Sigma(E(u)))=0 \quad \text { in } \Omega \\
& -(\mathbf{1}+\nabla u) \Sigma(E(u)) \cdot \nu+g(u)=0 \quad \text { on } \partial \Omega,
\end{aligned}
$$

where $\Sigma$ is a tensor defined by

$$
\Sigma(E)=\lambda \operatorname{Tr}(E) \mathbf{1}+2 \mu E
$$

and $g$ represents the applied surface forces. It can be computed from the norm of the image gradient, an edge map obtained using a Canny operator [2] smoothed with a Gaussian filter, or a distance map [1], [15]. The Gradient Vector Flow algorithm (GVF) introduced in [18] generates a force field by an iterative diffusion process, which is not derived from a potential. Although not conservative, we observed that the force field obtained by the GVF method conducted to reasonable numerical results as compared to more conventional gradient based techniques.

\section{Approximation of the model using an incremental method}

The problem (6) can be written as

$$
L(u)=f(u) \text { in } \Omega \times \partial \Omega,
$$

where

$$
L(u)=\left(\begin{array}{c}
\operatorname{div}((\mathbf{1}+\nabla u) \Sigma(E(u))) \\
\mathbf{1}+\nabla u) \Sigma(E(u)) \cdot \nu
\end{array}\right), \quad f(u)=\left(\begin{array}{c}
0 \\
g(u)
\end{array}\right) .
$$

To solve problem (8), an incremental method is proposed, which consists in letting the forces vary by small increments from 0 to their calculated value and computing corresponding approximate solutions by successive linearizations. For $\lambda \in[0,1]$, define $u(\lambda)$ as the solution to

$$
L(u(\lambda))=\lambda f(u(\lambda)) \quad \text { in } \Omega \times \partial \Omega
$$

After differentiating this relation with respect to $\lambda$ and adding an initial condition, we obtain

$$
\begin{aligned}
& u^{\prime}(\lambda)=\left(L^{\prime}(u(\lambda))-\lambda f^{\prime}(u(\lambda))\right)^{-1} f(u(\lambda)), 0 \leq \lambda \leq 1, \\
& u(0)=0 .
\end{aligned}
$$

Note that, if $u$ is a solution of the differential equation (10), by integration of this equation, $u$ is also solution of equation (9). Therefore, the study of the partial differential equations (9) reduces to solving the ordinary differential equation (10). In [14], sufficient conditions for the convergence of the Euler's algorithm are given for the problem (10). In next sections, this incremental method is applied to image segmentation. 


\section{Finite Element Discretization}

For the numerical optimization of the total energy (6) with the incremental method presented in the previous section, the finite element method is used. Let $M$ be a positive number, $\left\{\psi_{1}, \cdots, \psi_{M}\right\}$ a function basis for the approximation of the displacements. The algorithm is detailed below:

Let $U_{0}$ a vector containing displacement components.

- Initialization step: $U_{0}=0$ and $u_{0}=0$;

- Iterations

(i) Assemble the stiffness matrix at iteration $n$

$$
\mathcal{K}_{i j}^{n}=\int_{\Omega_{h}} k\left(u_{n}, \psi_{i}, \lambda_{n}\right): \nabla \phi_{j} \mathrm{~d} x-\left\langle g^{\prime}\left(u_{n}\right) \cdot \psi_{i}, \psi_{j}\right\rangle, \quad 1 \leq i, j \leq M
$$

(ii) Solve the linear system, with $U_{n+1}$ as unknown

$$
\mathcal{K}^{n} U_{n+1}=\mathcal{K}^{n} U_{n}+\left(\lambda_{n+1}-\lambda_{n}\right) \mathcal{F}\left(U_{n}\right) ;
$$

(iii) Compute the approximate displacement at iteration $n+1$

$$
u_{n+1}=\sum_{i=1}^{i=M} U_{n+1, i} \psi_{i} ;
$$

(iv) If $\left\|u_{n+1}-u_{n}\right\|<\epsilon$ stop, otherwise go to (i).

Where $\mathcal{F}$ has the components:

$$
\mathcal{F}_{j}\left(U_{n}\right)=<g\left(u_{n}\right), \psi_{j}>, 1 \leq j \leq M
$$

and

$$
k(u, w)=(\mathbf{1}+\nabla u) \Sigma\left(\epsilon(w)+\frac{1}{2}\left(\nabla u^{t} \nabla w+\nabla w^{t} \nabla u\right)\right)+\nabla w \Sigma(\mathbf{E}(u)) .
$$

The scalar product $\langle\cdot, \cdot\rangle$ is defined on $\partial \Omega$ for the two functions $\alpha$ and $\beta$ by :

$$
<\alpha, \beta>=\int_{\partial \Omega} \alpha(x) \beta(x) \mathrm{d} \sigma .
$$

The stiffness matrix $\mathcal{K}^{n}$ can be updated at each iteration, but this is quite time consuming. The convergence of the algorithm can be accelerated by updating the stiffness matrix $\mathcal{K}^{n}$ after several iterations instead of updating it at each iteration. Two types of iterations are thus used: internal and external iterations. With the external iterations, the stiffness matrix is updated, which permits large displacements. Internal iterations do not require the computation of the matrix. Hence, less computing time is needed for internal iterations than for external iterations. The number of external iterations should therefore be reduced as much as possible but depends on the problem. If only small displacements are required it is not necessary to update the stiffness matrix. In that case, internal iterations suffice. 


\section{Simultaneous Segmentation of the left and right Heart Ventricles in 3D Cine MR Images}

\subsection{Experimental data}

Mouse heart cine MR images were acquired with a $7 \mathrm{~T}$ magnetic resonance scanner with a whole body coil for RF excitation and a $15 \mathrm{~mm}$ surface coil for MR signal reception. An ECG-gated FLASH sequence was used to acquire short-axis cine images with $25 \mathrm{~mm}^{2} \mathrm{FOV}, 256 \times 256$ pixels, $1 \mathrm{~mm}$ slice thickness, $7 / 3.5 \mathrm{~ms}$ TR/TE, $64 \mathrm{KHz}$ bandwidth and 20 degrees flip-angles. Cine images (16 frames over the cardiac cycle) were acquired for 7 slice levels, covering the entire left ventricle $(\mathrm{LV})$. With a cardiac frequency of 450 b.p.m, the total acquisition time was 20 minutes.

\subsection{Image data preprocessing and initial model placement}

High resolution MR images have a relatively low SNR. Furthermore, the abundance of small features in the images (papillary muscles, coronaries, etc.) can lead to local minima of the model energy, leading to inaccurate segmentation. Both problems need to be addressed before the image edges can be extracted. We applied morphological operators to remove small features and noise while preserving strong edges (see [16]).

The non rigid segmentation algorithm requires that the model be initialized close to the targeted object, in this case the mouse heart. Various solutions have been proposed to solve this problem. A fully automatic approach can be used, optimizing an affine transformation to match two criteria: distance to the closest edge, and appearance. In [8], the Powell optimization method is used to minimize the resulting energy. Results can be further improved by repeating the Powell optimization with several random initializations and keeping the result with the lowest energy, thus reducing the risk of falling into a local minimum.

The fully automated method was found to yield generally good results, however it is not always robust. In order to give more reproducible results, a semiautomatic approach was preferred here. In this approach, a medical expert selects several fiducial points located on the heart contours, both on the heart model and in the acquired images. This information is used to compute the best affine registration using a least squares approach. Registration can be further refined by adding a thin-plate spline registration step with the same fiducial points. More details on this process can be found in [17].

\subsection{Results}

Four 4D MRI sequences corresponding to 4 different mice were processed using our method. The model parameters were set to: Young modulus of $10 \mathrm{~Pa}$ for the LV and $40 \mathrm{~Pa}$ for the right ventricle (RV), and a Poisson coefficient of 0.485 for the whole model. Figure 1 illustrates the $3 \mathrm{D}$ segmentation process. Figure 2-(a)(b) shows the results of the deformation of the 3D mesh for the same 3D image. 
Results show that although we are still experiencing a few specific problems, our method is able to correctly locate the heart borders in the images.

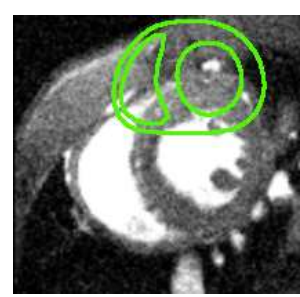

(a)

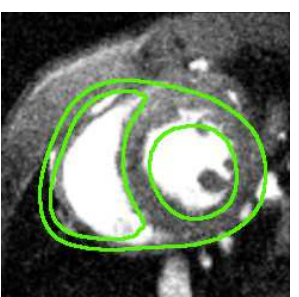

(b)

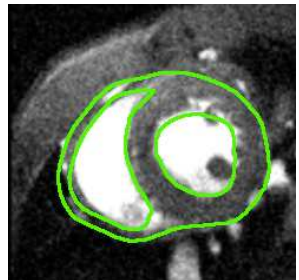

(c)

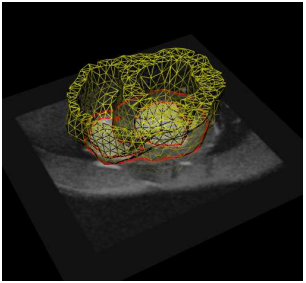

(d)

Fig. 1. Segmentation of 3D mouse MR data : (a) Initial positioning of the DET model, (b) Result of affine positioning, (c) Segmentation result, and (d) 3D mesh obtained as a result of the segmentation process

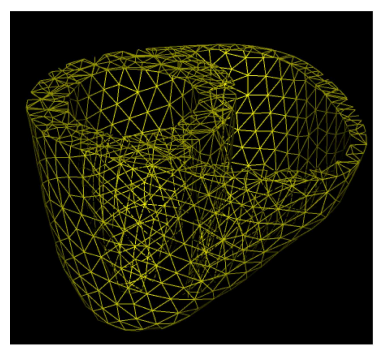

(a)

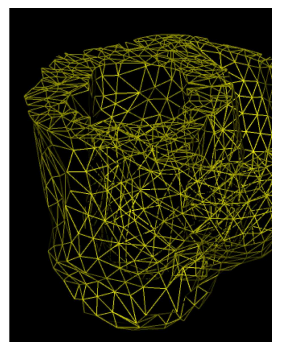

(b)

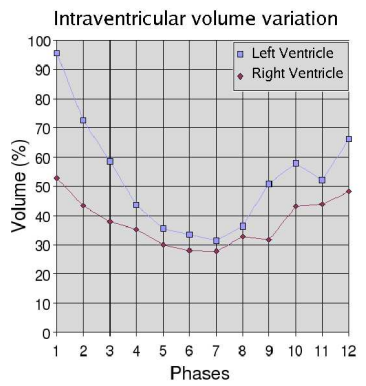

Phases

Fig. 2. (a) Original model (b) deformed model; Diagram on the right: Estimated volume variation of the ventricles over the cardiac cycle.

\subsection{Segmentation over the cardiac cycle}

Segmentation tracking of the heart is achieved by taking the segmentation result at the present time frame as the initial solution for the next time frame, and repeating the process for all the times frames in the MRI sequence. Once the $3 \mathrm{D}$ contours have been extracted, it is straightforward to compute the enclosed ventricle volumes. Figure 2 shows on the right an example of volume variation curves obtained by automatic segmentation tracking. The overall volume variation pattern is coherent. However, localized problems persist during early diastole. These problems may be tackled by incorporating temporal constraints for the tracking of the heart shape over time. 


\section{Discussion and conclusions}

This article presents the non linear deformable elastic template model for the segmentation of soft structures in image sequences. One striking feature of this model is that convergence results for the incremental method, which is used to approximate a solution for a 3D nonlinear elastic template under successive forces, are available. This sets a convenient framework for the segmentation of soft structures in $3 \mathrm{D}$ and $3 \mathrm{D}+$ time images.

The proposed model has been experimented here for the segmentation of MR image sequences of mouse hearts. The proposed method was able to retrieve the heart contours in most cases, allowing the computation of volume variation curves. However, manual interactions and corrections of the results would still be needed to use the method routinely. Remaining problems include inaccuracies in the segmentation of the pericardium due to the presence of numerous anatomical structures close to the heart, and localized errors during the early diastolic phase due to motion artifacts.

Note that for the segmentation over a cardiac cycle, progressive segmentation (see section 6.4) was used. It would be interesting to include a temporal constraint in the model by introducing non stationary equations for the segmentation over the cardiac cycle. Larger scale experiments, including quantitative evaluation of the segmentation accuracy is also be needed to fully validate the method.

\section{Acknowledgments:}

We thank Prof. M. Schatzman for interesting discussions and her constant support. We are also very grateful to B. Hiba and M. Janier for providing the MR images of the mice. The Région Rhônes-Alpes is gratefully acknowledged for its support through the project PP3-I3M of the cluster ISLE. This work is partially funded by the french ACI-AGIR project (http://www.aci-agir.org/).

\section{References}

1. G. Borgefors. Distance transformation in digital images. Computer Vision Graphics and Image Processing, 48:344-371, 1986.

2. John F. Canny. A computational approach to edge detection. IEEE Transactions on Pattern Analysis and Machine Intelligence, 8:679-698, 1986.

3. Philippe G. Ciarlet. Mathematical elasticity. Vol. I, volume 20 of Studies in Mathematics and its Applications. North-Holland Publishing Co., Amsterdam, 1988.

4. A. F. Frangi, D. Rueckert, J. A. Schnabel, and Niessen W. J. Automatic construction of multiple-object three-dimensional statistical shape models. IEEE Transactions on Medical Imaging, 21(9):1151-1166, 2002.

5. Michael R. Kaus, Jens von Berg, Jürgen Weese, Wiro Niessen, and Vladimir Pekar. Automated segmentation of the left ventricle in cardiac MRI. Medical Image Analysis, 8:245-254, 2004.

6. Gregory. J. Klein and Ronald H. Huesman. Four-dimensional processing of deformable cardiac PET data. Medical Image Analysis, 6:29-46, 2002. 
7. Jyrki Lötjönen, S. Kivistö, J. Koikkalainen, D. Smutek, and Kirsi Lauerma. Statistical shape model of atria, ventricles and epicardium from short- and long-axis MR images. Medical Image Analysis, 8:371-386, 2004.

8. Timo Mäkelä, Quoc Cuong Pham, Patrick Clarysse, Jukka Nenonen, Jyrki Lötjönen, Outi Sipilä, Helena Hänninen, Kirsi Lauerma, Juhani Knuuti, Toivo Katila, and Isabelle E. Magnin. A 3-D model-based registration approach for the PET, MR and MCG cardiac data fusion. Medical Image Analysis, 7:377-389, 2003.

9. Steven C. Mitchell, Johan G. Bosch, Boudewijn P. F. Lelieveldt, Rob J. van der Geest, J. H. C. Reiber, and Milan Sonka. 3D active appearance models: segmentation of cardiac MR and ultrasound images. IEEE Transactions on Medical Imaging, 21(9):1167-1178, 2002.

10. J. Montagnat and H. Delingette. A review of deformable surfaces: topology, geometry and deformation. Image and Vision Computing, 19(14):1023-1040, 2001.

11. Stanley Osher and James A. Sethian. Fronts propagating with curvature-dependent speed: algorithms based on hamilton-jacobi formulations. J. Comput. Phys., 79(1):12-49, 1988.

12. Q.-C. Pham, F. Vincent, P. Clarysse, P. Croisille, and I. E. Magnin. A FEMbased deformable model for the 3D segmentation and tracking of the heart in cardiac MRI. In 2nd International Symposium on Image and Signal Processing and Analysis (ISPA 2001), volume 1, pages 250-254, Pula, Croatia, 2001.

13. M. Picq, J. Pousin, and Y. Rouchdy. A Linear 3D Elastic Segmentation Model for Vector Fields. Application to the Heart segmentation in MRI. To be published in Journal of Mathematical Imaging and Vision, 2007.

14. Y. Rouchdy, J. Pousin, J. Schaerer, and P. Clarysse. A nonlinear elastic deformable template for soft structure segmentation. Application to the heart segmentation in MRI. Accepted in Inverse Problems, 2007.

15. T. Saito and T.I. Toriwaki. New algorithm for euclidean distance transformation of an n-dimensional digitized picture with applications. Pattern recognition, 27:15511565, 1994.

16. J Schaerer, Y Rouchdy, P Clarysse, B Hiba, P Croisille, J Pousin, and IE Magnin. Simultaneous segmentation of the left and right heart ventricles in $3 \mathrm{D}$ cine MR images of small animals. In Proceedings of Computers in Cardiology, pages 231-234, Lyon, 2005.

17. Joël Schaerer, Zhen Qian, Patrick Clarysse, Dimitris Metaxas, Leon Axel, and Isabelle E. Magnin. Fast and automated creation of patient-specific $3 \mathrm{~d}$ heart models from tagged mri. In Proceedings of the MICCAI 2006 SA2PM Workshop.

18. C. Xu and J.L. Prince. Snakes, shapes and gradient vector flow. IEEE Trans. Image Processing, 7:359-369, 1998. 